Agronomía Costarricense 43(1): 9-19. ISSN:0377-9424 / 2019

www.mag.go.cr/rev_agr/index.html www.cia.ucr.ac.cr

\title{
INCIDENCIA Y SEVERIDAD DE Olivea tectonae Y Rhabdopterus sp. EN PLANTACIONES JÓVENES DE Tectona grandis L.f. BAJO DISTINTAS MODALIDADES DE CONTROL DE ARVENSES
}

\author{
Marcela Arguedas-Gamboa ${ }^{1 / *}$, María Rodríguez-Solís*, Mario Guevara-Bonilla*, \\ Edwin Esquivel-Segura*, Simón Sandoval-Rocha ${ }^{* *}$,Elemer Briceño-Elizondo*
}

Palabras clave: Cultivos de cobertura; teca; plagas y enfermedades; control de malezas.

Keywords: Cover crops; teak; pest and diseases; weed control.

Recibido: $13 / 04 / 18$

Aceptado: 23/07/18

RESUMEN

Tectona grandis L.f. (teca) es la especie forestal con fines comerciales más cultivada en Costa Rica. Dentro de las actividades de manejo de estas plantaciones se encuentran el control de arvenses y el manejo integrado de plagas y enfermedades. Recientemente se han utilizado cultivos de cobertura como método alternativo de control de arvenses, sin embargo, se desconoce la afectación de este control en las plagas. Se desarrolló una investigación para determinar el efecto de la utilización de cultivos de cobertura en el control de arvenses y evaluar la presencia de herbívoros y organismos fitopatógenos en plantaciones jóvenes de teca en el Pacífico Sur de Costa Rica. Se utilizó un diseño experimental de bloques completos al azar con 3 repeticiones y 7 tratamientos: Testigo (sin control de arvenses), Control de arvenses químico, Control de arvenses manual, Canavalia ensiformis, Vigna radiata, Mezcla entre Vigna radiata y Pueraria phaseoloides y Crotalaria juncea. Se seleccionaron como enfermdad objetivo Olivea tectonae ("roya de la teca") y plaga objetivo Rhabdopterus sp. ("esqueletizador de la teca") y se realizaron 2 evaluaciones de incidencia e índice promedio

1 Autora para correspondencia. Correo electrónico: marguedas@itcr.ac.cr

\section{ABSTRACT}

Incidence and severity of Olivea tectonae and Rhabdopterus sp. in young plantations of Tectona grandis L.f. under different modalities of weed control. Tectona grandis L.f. (teak) is the most cultivated precious wood forest species in the world and in Costa Rica it has been used in commercial reforestation projects. Among the silvicultural activities of these plantations are weed and the prevention and management of pests. Recently, cover crops have been used as an alternative method of weed control, however, the effect of this control on pests is unknown. A study was developed to determine the effect of the use of cover crops in the control of weeds and to evaluate the presence of herbivores and pathogens in young teak stands in the South Pacific of Costa Rica A randomized complete block design with 3 replications and 7 treatments was used: Control (without weed control), Chemical weed control, Manual weed control, Cover crop Canavalia ensiformis, Cover crop Vigna radiata, Mix between Vigna radiata and Pueraria phaseoloides and Cover crop Crotalaria juncea. Olivea tectonae ("teak rust") and Rhabdopterus $s p$. ("Teak skeletonizer") were selected as target

\footnotetext{
* Instituto Tecnológico de Costa Rica (ITCR), Escuela de Ingeniería Forestal, Costa Rica.

** Bioforest, Chile.
} 
de severidad, cuando la plantación tenía 6 y 12 meses de edad. A pesar de que la incidencia mostró valores superiores a $60 \%$ en ambas los valores promedios de severidad se concentraron en las categorías menores. Adicionalmente en las evaluaciones realizadas no se evidenció efecto de las coberturas en el aumento de la incidencia o severidad de la enfermedad y plaga evaluadas, lo que demuestra que el uso de coberturas puede ser una alternativa viable para el control de arvenses de una plantación de teca. Por lo anterior se recomienda continuar con estudios que evalúen el efecto del uso de coberturas en la presencia y severidad de plagas en teca en distintas regiones del país.

\section{INTRODUCCIÓN}

Tectona grandis L.f. (teca), es la especie forestal de madera preciosa más cultivada en el mundo. Es una especie de fácil propagación, establecimiento y manejo; su madera de excelente calidad es muy apreciada para la construcción de muebles, elementos estructurales, embarcaciones, carpintería, chapas, pisos, entre otros (Keogh 2013, Moya et al. 2014). El cultivo de la teca es un recurso emergente con grandes posibilidades en América Latina (de Camino y Morales 2013, Kollert y Kleine 2017). En Costa Rica se ha utilizado en proyectos de reforestación con fines comerciales (Montagnini 2004, Boley et al. 2009) y, hasta el 2014, el Instituto Nacional de Estadística y Censo (INEC) informa de 47167 ha plantadas con esta especie y más de 150000 árboles dispersos en fincas (INEC 2015).

Con respecto a las plagas, para América Tropical, Arguedas et al. (2013) reportan 53 especies de insectos, 34 de patógenos, 2 vertebrados y 4 muérdagos que afectan $T$. grandis. En plantaciones recién establecidas, las plagas pueden causar disminución del área foliar y, por ende, disminución del crecimiento. Entre disease and pest where 2 evaluations of the incidence and severity index were made, when the plantation was 6 and 12 months old. Despite the fact that incidence values were higher than $60 \%$ in both, the average values of severity were grouped in the minor categories. In addition, no significant differences were found among treatments and the incidence or severity of the evaluated pests, which demonstrates that the use of cover crops can be a viable alternative for the control of weeds in a teak plantation. Therefore, we recommend to continue with studies that evaluate the effect of the use of coverages in the presence and severity of teak pests in different regions of the country.

las principales plagas causantes de estos daños se encuentran el Rhabdopterus sp. (Coleoptera, Chrysomelidae, Eumolpinae) y la roya de la teca, causada por el patógeno Olivea tectonae (T.S. Ramakr. \& K. Ramakr.) J.L. Mulder.

Las especies de Rhabdopterus son polífagas; los adultos se alimentan de follaje de $T$. grandis, por lo que producen perforaciones características en forma elongada y curva (Muñoz 2002, Arguedas et al. 2013). Estas especies poseen hábitos alimenticios nocturnos y los ataques están relacionados con la dominancia de gramíneas en el sitio, ya que las larvas se alimentan de sus raíces (Arguedas et al. 2013).

Por otra parte, Olivea tectonae afecta el follaje de la teca desde su estado de plántulas en el vivero hasta árboles adultos. En estos últimos, las hojas afectadas son las más viejas, especialmente las de las partes bajas. Las afectaciones pueden provocar deshoja prematura y reducción de las tasas de crecimiento esperadas (Arguedas 2004, Arguedas et al. 2006, Arguedas et al. 2013). Esta enfermedad ha sido reportada en toda Asia; sin embargo, durante los últimos años se ha presentado en plantaciones de $T$. grandis 
en América desde el sur de México hasta Ecuador, Brasil y el Caribe (Matarrita et al. 2006, Pérez et al. 2008, Cabral et al. 2010, EPPO 2005, Mesquita et al. 2016).

Hay muchas actividades dentro del manejo de las plantaciones forestales que deben ser contempladas para garantizar el éxito de las mismas. Entre ellas se encuentran el control de malezas o plantas arvenses, así como la prevención y manejo de plagas. Las malezas constituyen uno de los mayores impedimentos para el establecimiento exitoso (supervivencia), crecimiento inicial y productividad de plantaciones forestales (Adams et al. 2003, Garau et al. 2009, Ladrach 2010). Adicionalmente, podrían ser hospederos de plagas y enfermedades que afecten al crecimiento de los árboles.

Tradicionalmente en Costa Rica, en plantaciones forestales, se han realizado el control de arvenses de forma manual y mediante control químico con herbicidas (Guevara 2011), pero, el alto costo y los potenciales efectos negativos en la salud humana y el medio ambiente (Jabran et al. 2015, FSC 2015) han generado que se busquen nuevas alternativas de control.

A nivel mundial y principalmente en la agricultura, los cultivos de cobertura se han convertido en uno de los métodos alternativos de control de arvenses y de remplazo de herbicidas más importantes y su manejo puede proveer numerosos servicios ecosistémicos, que incluyen además, del mejoramiento de la calidad de los suelos, el aumento de la productividad del cultivo principal y la regulación de plagas (Lundgren y Fergen 2011, Schipanski et al. 2014).

La práctica del uso de cultivos de cobertura ha sido muy utilizada en la agricultura, sin embargo, son mínimas las experiencias del uso de coberturas en plantaciones forestales tropicales y su relación con la severidad de daños por plagas es desconocida. Es por ello que el presente trabajo pretende determinar el efecto de la utilización de cultivos de cobertura en la presencia de herbívoros y organismos fitopatógenos en plantaciones jóvenes de $T$. grandis en la zona Pacífico Sur de Costa Rica, provincia de Puntarenas.

\section{MATERIALES Y MÉTODOS}

\section{Área de estudio}

El ensayo se realizó en la localidad de Salamá, Provincia de Puntarenas, Costa Rica ( $8^{\circ} 48^{\prime} 41^{\prime \prime} \mathrm{N}$ y $83^{\circ} 17^{\prime} 37^{\prime \prime}$ O) a una altitud de $20 \mathrm{~m}$. La zona de vida, de acuerdo con Holdridge (1987), es bosque muy húmedo tropical, con una temperatura promedio de $26^{\circ} \mathrm{C}$ y una precipitación media anual de $4450 \mathrm{~mm}$. El suelo de este sitio es de orden Inceptisol según la clasificación USDA (2003).

\section{Preparación del terreno y siembra de coberturas}

Antes de establecer los sitios de ensayo, se mecanizó el terreno utilizando un tractor de 95 caballos de fuerza, un arado de cincel y una rastra de 16 discos. Posteriormente a la mecanización inicial, se establecieron camellones de $30 \mathrm{~cm}$ de altura en donde fueron plantados los árboles con un distanciamiento de 3,1 x 3,1 m. El área de ensayo estuvo libre de arvenses al momento de la siembra de las coberturas.

Los cultivos de cobertura fueron sembrados 15 días después de establecida la plantación. El hoyado y la siembra se realizó de forma manual a una distancia de $50 \mathrm{~cm}$ entre hoyos y filas.

\section{Selección de las plagas evaluadas}

Con el fin de evaluar el estado fitosanitario de las plántulas y seleccionar las plagas "objetivo" para la valoración de herbivoría, se realizó una valoración cualitativa al ensayo a los 3 meses de establecido. Para ello, en cada unidad experimental, se seleccionaron y se recorrieron 2 transeptos correspondientes a las filas 2 y 3 (16 árboles). Se identificaron los problemas fitosanitarios por medio de los síntomas para el caso de las enfermedades y los daños para las plagas insectiles (según las descripciones de Arguedas et al. 2013). Para cada unidad experimental, los síntomas y los daños se valoraron en: severo $(40,1-$ $60 \%$ de follaje afectado), moderado (20,1-40 de follaje afectado), leve (0,1-20 de follaje afectado) 
y esporádico (casos muy aislados), puntuados respectivamente en 4, 3, 2 y 1 . Se sumaron los puntajes para las 21 unidades experimentales y se seleccionaron los 2 de mayor puntaje.

\section{Valoración de incidencia y severidad}

Se realizaron 2 evaluaciones de las plagas objetivo, la primera cuando la plantación tenía 6 meses de establecida (diciembre de 2016) y la segunda a los 12 meses (junio de 2017).

En cada unidad experimental se determinó el porcentaje de incidencia (I) (ecuación 1) y el índice promedio de la severidad (IPS) de la enfermedad (ecuación 2), acorde con las metodologías propuestas por Arauz (1998) y Couto et al. (2007).

$$
I=\frac{\text { Total de árboles enfermos }}{\text { Total de árboles muestreados }} * 100
$$

$$
I P S=\frac{\sum(\text { Grado de Categoría } * \text { Frecuencia })}{\text { Total de unidades evaluadas }} * 100
$$

La incidencia indica la cantidad de árboles enfermos con respecto a la totalidad de árboles evaluados, la severidad evalúa la frecuencia con la que se presentan las diferentes categorías de daño en relación con el total de árboles enfermos. Para este ensayo, se utilizaron 5 categorías de severidad respecto al porcentaje de follaje afectado por cada una de las 2 plagas (Cuadro 1).

Cuadro 1. Categorías de severidad para la evaluación de los daños producidos por Rhabdopterus sp. y Olivea tectonae en Tectona grandis.

\begin{tabular}{cc}
\hline Categoría de severidad & Follaje afectado $(\%)$ \\
\hline A & $0,1-20,0$ \\
B & $21,0-40,0$ \\
C & $41,0-60,0$ \\
D & $61,0-80,0$ \\
E & $81,0-100,0$ \\
\hline
\end{tabular}

\section{Diseño experimental}

Se utilizó un diseño experimental de bloques completos al azar con 3 repeticiones y 7 tratamientos. Los tratamientos aplicados fueron: T0: Testigo (sin control de malezas), T1: Control de malezas químico, T2: Control de malezas manual, T3: Cultivo de cobertura Canavalia ensiformis, T4: Cultivo de cobertura Vigna radiata, T5: Mezcla entre Vigna radiata y Pueraria phaseoloides y T6: Cultivo de cobertura Crotalaria sp. Cada unidad experimental estuvo conformada por 60 árboles incluyendo los bordes. La parcela efectiva de medición fue de 40 árboles distribuidos en 4 filas con 10 árboles cada una.

\section{Análisis estadístico}

El efecto de los tratamientos y la fecha de medición sobre las variables de severidad fueron analizadas mediante modelos lineales generalizados, en el que se solucionó el problema del nocumplimiento de normalidad de los errores. Esto es, debido a que el índice de severidad se calculó de conteos agrupados en 5 categorías. En esos modelos, se evaluaron 4 diferentes distribuciones (Normal, Poisson, Gamma y Geométrica), para buscar el mejor ajuste del modelo que permitiera hacer inferencia sobre el efecto de los tratamientos. Para el caso de las variables de incidencia, se realizó un análisis de varianza bajo los supuestos de normalidad en un diseño en bloques aleatorios. Todos los análisis se realizaron con el software estadístico SAS Versión 9.1 (SAS-INSTITUTE-INC 2000).

\section{RESULTADOS}

\section{Selección de las plagas objetivo}

Las 2 plagas con mayor puntaje obtenido fueron Olivea tectonae y Rhabdopterus sp., con 41 y 23 puntos respectivamente; por lo tanto, el análisis de incidencia y severidad se realizó con base en estas 2 plagas objetivo. Adicionalmente, 
se reportaron síntomas de Cercospora sp. y daños de Atta spp., Spodoptera sp., Walteraniella sp., Aphis sp., Oxidia sp. y varias especies del orden Orthoptera, con puntajes entre 9 y 3 .

Las hojas de teca afectadas por Olivea tectonae presentan inicialmente manchas necróticas de tamaño y forma variable, de color verdoso que cambia a tonos claros y luego a café y grises
(Figura 1A). En el envés se forman estructuras llamadas uredinios, los cuales son eruptivos, cilíndricos y curvados; su abundancia es tan grande que las esporas o urediniosporas que liberan cubren toda la superficie inferior de la hoja, que le dan un color anaranjado y una apariencia polvosa (Figura 1B) (CABI 2017, Cibrián y Arguedas 2007).
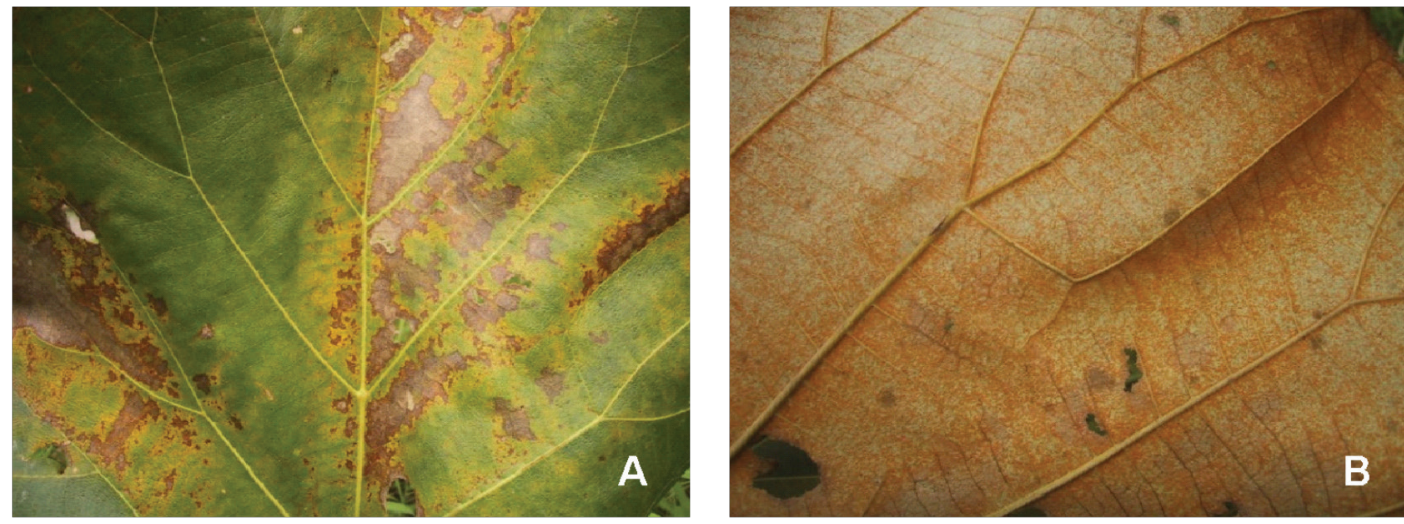

Fig. 1. Olivea tectonae en Tectona grandis L.f. Puntarenas, Costa Rica. A: Síntomas necróticos en el follaje, B: masas de urediniosporas en el envés de la hoja. Puntarenas, Costa Rica.

Los adultos de Rhabdopterus realizan perforaciones en el follaje de teca de forma elongada y curva de aproximadamente $1,3 \mathrm{~cm}$ de largo y 0,16 cm de ancho (Figura 2) (Muñoz 2002, Arguedas et al. 2013).

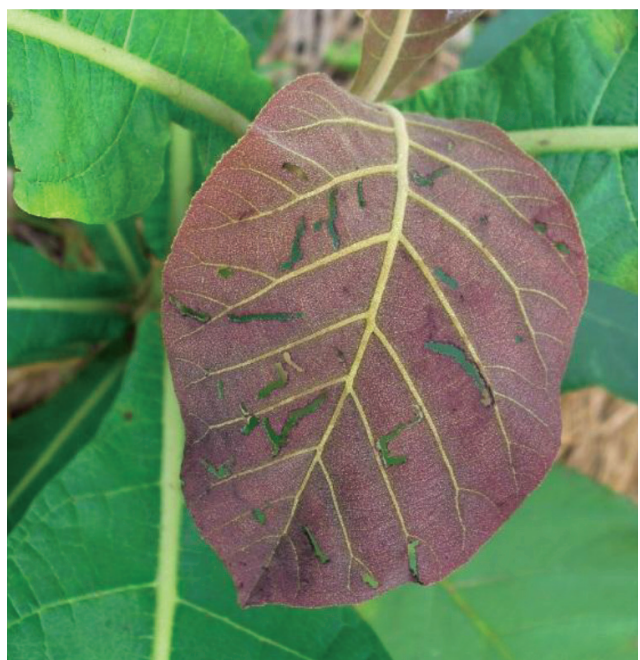

Fig. 2. Hoja joven de Tectona grandis con perforaciones de herbivoría provocadas por adultos de Rhabdopterus sp. Puntarenas, Costa Rica. 


\section{Evaluación de incidencia y severidad}

Para el ataque de $O$. Tectonae, se demostró que es un patógeno que puede afectar a su hospedero en etapas juveniles (entre 6 y 12 meses de edad), como lo indican Arguedas et al. (2013) para la región neotropical americana. En ambas mediciones (diciembre de 2016 y junio de 2017), en la Mitad de la Copa Inferior (MCI), la incidencia osciló entre 90 - 100\%, mientras que en la Mitad de la Copa Superior (MCS), la incidencia osciló entre 0-29\%. Para los parámetros de medición de severidad en MCS, los valores de la segunda medición (junio del 2017) son iguales o inferiores a los de la primera medición (diciembre del 2016), debido a que los árboles más jóvenes son más susceptibles a la enfermedad. En el caso de la severidad en MCI, sucede lo contrario, los valores de la segunda medición son superiores a los de la primera, lo que indica que la severidad del ataque aumentó con el tiempo y en mayor proporción en las hojas maduras.

Además, a excepción del parámetro de Incidencia de la MCI para la medición de diciembre del 2016, el tratamiento "Mezcla" fue el más bajo para las otras condiciones de Incidencia (Figura 3). Sin embargo, no se encontraron diferencias significativas entre tratamientos para ninguna de las variables evaluadas (Cuadro 2). Solo se encontraron diferencias por fecha de medición para las 2 partes de la copa (MCI y MCS).
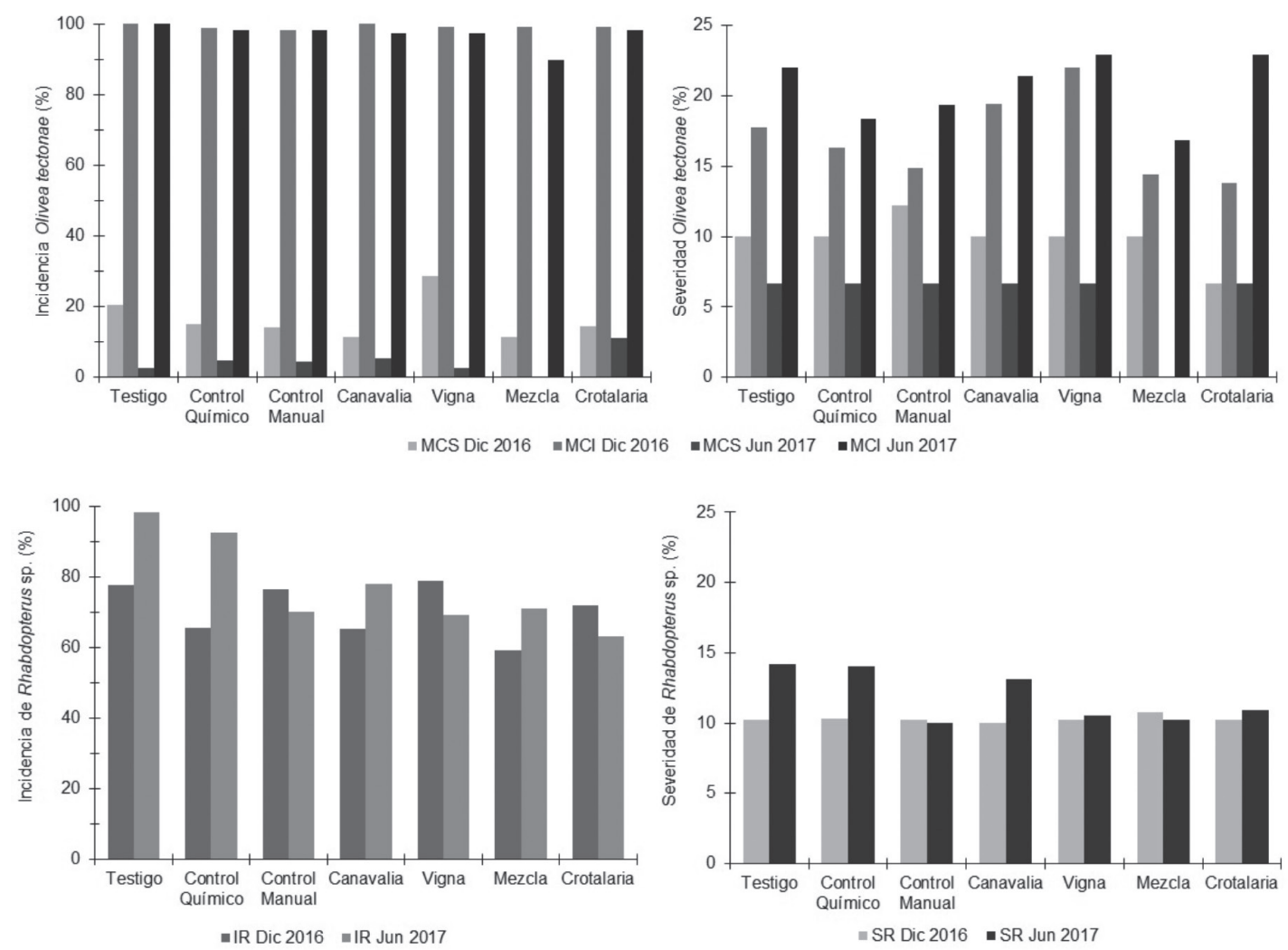

Nota: MCI, Mitad Copa Inferior; MCS, Mitad Copa Superior; IR, Incidencia Rhabdopterus sp.; SR, Severidad Rhabdopterus sp.

Fig. 3. Incidencia y severidad promedio de Olivea tectonae y Rhabdopterus sp. en plantaciones de Tectona grandis bajo distintos métodos de control de arvenses. Puntarenas, Costa Rica. 
Cuadro 2. Significancia de factores para la severidad e incidencia de los agentes Olivea tectonae y Rhadbopterus sp. evaluado en la copa de Tectona grandis.

\begin{tabular}{|c|c|c|c|c|c|}
\hline $\begin{array}{c}\text { Tipo de } \\
\text { evaluación }\end{array}$ & Agente & $\begin{array}{c}\text { Porción de la } \\
\text { copa }\end{array}$ & $\begin{array}{c}\text { Distribución } \\
\text { de variable }\end{array}$ & Factor & Probabilidad \\
\hline \multirow{6}{*}{$\begin{array}{l}\text { Severidad } \\
\quad \text { (IPS) }\end{array}$} & \multirow{4}{*}{ Olivea tectonae } & \multirow{2}{*}{ MCS } & \multirow{2}{*}{ Poisson } & Fecha & $<0,0001$ \\
\hline & & & & Tratamiento & 0,0961 \\
\hline & & \multirow{2}{*}{ MCI } & \multirow{2}{*}{ Gamma } & Fecha & 0,0167 \\
\hline & & & & Tratamiento & 0,2463 \\
\hline & \multirow{2}{*}{ Rhabdopterus sp. } & & \multirow{2}{*}{ Poisson } & Fecha & 0,0013 \\
\hline & & & & Tratamiento & 0,0829 \\
\hline \multirow{6}{*}{ Incidencia (I) } & \multirow{4}{*}{ Olivea tectonae } & \multirow{2}{*}{ MCS } & \multirow{2}{*}{ Normal } & Fecha & 0,0027 \\
\hline & & & & Tratamiento & 0,8562 \\
\hline & & \multirow{2}{*}{ MCI } & \multirow{2}{*}{ Normal } & Fecha & 0,1406 \\
\hline & & & & Tratamiento & 0,5661 \\
\hline & \multirow{2}{*}{ Rhabdopterus sp. } & & \multirow{2}{*}{ Normal } & Fecha & 0,2423 \\
\hline & & & & Tratamiento & 0,4288 \\
\hline
\end{tabular}

Nota: los valores en "negrita” son significativos. MCI, Mitad Copa Inferior; MCS, Mitad Copa Superior.

Con respecto al ataque de Rhabdopterus sp., se observa que la severidad fue muy baja para todos los tratamientos en la primera medición, cuando los árboles tenían 6 meses, lo cual puede reducir los riesgos de muerte en el primer año de establecimiento. La incidencia y la severidad para las 2 mediciones (diciembre de 2016 y junio de 2017) y en todos los tratamientos varía entre 59,25-78,73\%, 63,26-98,33\%, $10,00-10,74 \%$ y 10,00 y 14,21 , respectivamente (Figura 3).

Por su parte, en la evaluación del efecto de los tratamientos, ninguno evidenció respuestas significantes en severidad e intensidad del ataque de los patógenos; sólo en algunos casos en la fecha de evaluación. Esto es en la variable Severidad de Olivea tectonae en MCS ( $\mathrm{p}<0,0001)$, Severidad de Olivea tectonae en MCI ( $\mathrm{p}=0,0167)$, Severidad de Rhabdopterus $\mathrm{sp}$. ( $\mathrm{p}=0,0013)$ e Incidencia de Olivea tectonae en MCS ( $\mathrm{p}=0,0027)$.

\section{Distribución por categorías de severidad}

La mayoría de los árboles afectados por el ataque de $O$. tectonae en la MCS se encontraron en la Categoría de Severidad A (Figura 4). A excepción sólo de la primera medición para el Control Manual, en que un 6\% de los árboles se encontraron en la Categoría de Severidad B y para la segunda medición para Mezcla que no había árboles enfermos. En la MCI se presentaron más árboles en otras categorías, pero los valores más altos se encontraron en la $\mathrm{A}$, cuyos porcentajes oscilaron entre $46 \%$ y $84 \%$; solamente para el Testigo (segunda medición) y para $V$. radiata (segunda medición), el porcentaje de árboles en la Categoría de Severidad B, es levemente superior al de la A (Figura 4). Además, se observó que, para todos los tratamientos en la segunda medición, el porcentaje de árboles en la Categoría de Severidad A siempre disminuyó, lo que indica que la severidad de la enfermedad aumentó en 6 meses. 

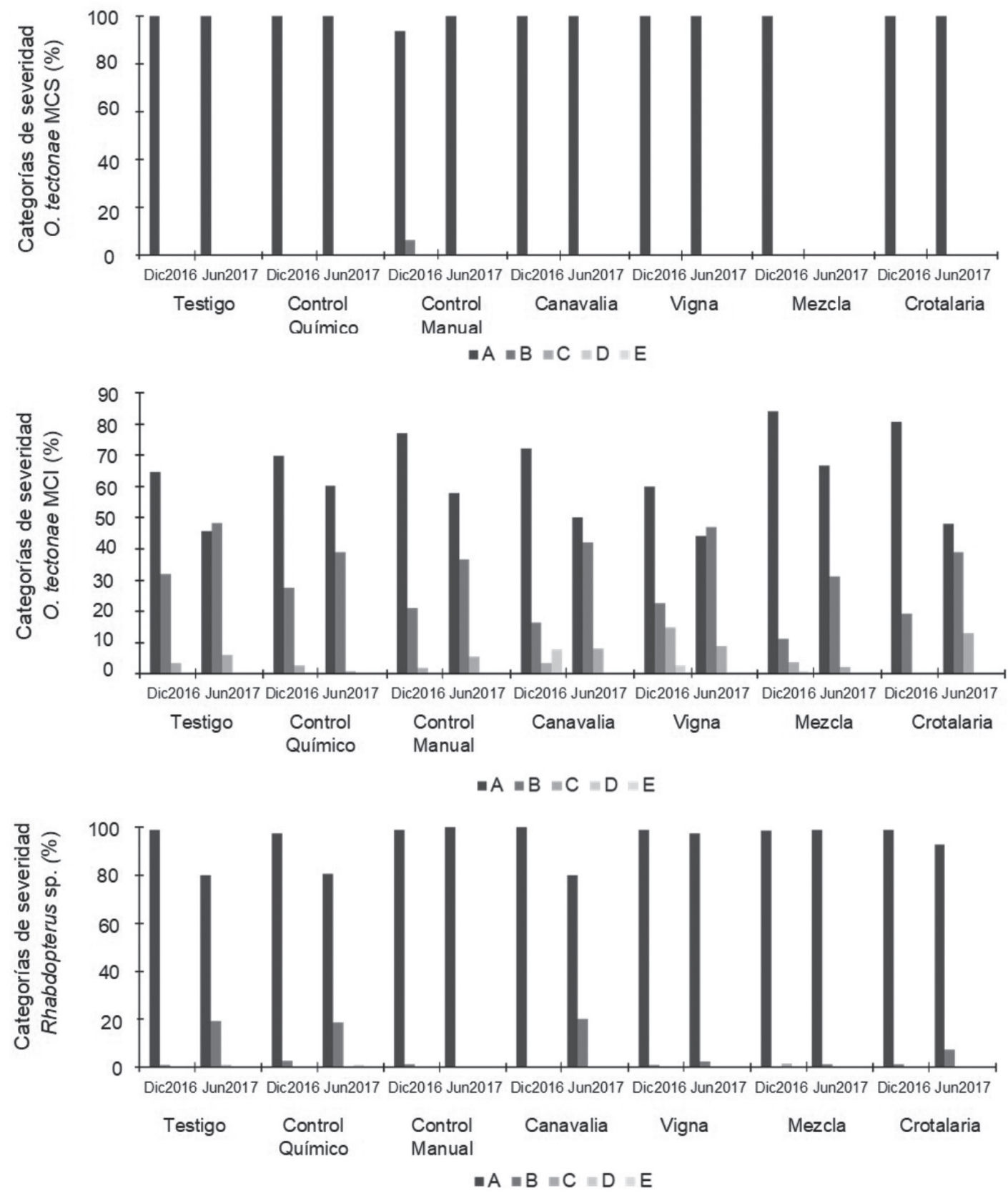

Fig. 4. Distribución por categorías de severidad de Olivea tectonae (MCI, Mitad Copa Inferior; MCS, Mitad Copa Superior) y Rhabdopterus sp. en plantaciones de Tectona grandis según tratamiento de coberturas y fecha de evaluación. Puntarenas, Costa Rica. 
Con respecto al ataque de Rhabdopterus sp., el comportamiento es similar al de O. tectonae MCS (Figura 4); el porcentaje para los diferentes tratamientos varió entre 80 y $100 \%$ para la Categoría de Severidad A, y entre 0 y $20 \%$ para la categoría B.

\section{DISCUSIÓN}

Las plagas seleccionadas como objetivo coinciden con los problemas fitosanitarios de mayor importancia encontrados por Baltodano (2008) en plantaciones jóvenes en Panamá, lo cual reduce potencialmente el crecimiento inicial de la Teca.

Los altos valores de incidencia encontrados para O. tectonae a los 6 meses de edad concuerdan con lo reportado con Sales et al. (2017) en plantaciones jóvenes en Brasil. Sin embargo, los resultados encontrados por estos autores fueron en plantaciones de 3 a 7 años de edad. Lo anterior indica que para las plantaciones de Teca la enfermedad es de rápida diseminación y avanza indistintamente de la cobertura presente en el suelo. Se confirma que ambas plagas se presentan en edades tempranas, lo que podría afectar el crecimiento, tal como reporta Arguedas et al. (2013). Además, diferencias entre las 2 partes de la copa evaluadas, sugieren que el no controlar la enfermedad podría aumentar los niveles de severidad del ataque sufrido.

Los cultivos de cobertura tienen el potencial de disminuir el uso de productos químicos tanto para el control de arvenses como para el control de plagas a través de un establecimiento en campo exitoso y promoviendo un incremento de enemigos naturales (Bone et al. 2009, Danne et al. 2010). No obstante, a nivel general, no se reportan estudios científicos del uso de coberturas vegetales en plantaciones forestales y hay escasa información sobre el efecto de cultivos de cobertura en el comportamiento de plagas en cultivos agrícolas.

Schipanski et al. (2014) indicaron que específicamente en el campo de las plagas existen incertidumbres en la estimación de los efectos de esta práctica en la dinámica insectos-plaga. Bachie (2011), en cultivos de hortalizas, tampoco encontró relación entre coberturas y el comportamiento de las plagas estudiadas, pero indica que promueven el crecimiento de poblaciones de parasitoides de dichas plagas.

Por su parte, Lundgren y Fergen (2011) indican que, en cultivos de maíz, las coberturas redujeron las poblaciones de la plaga subterránea Diabrotica virgifera (Coleóptera, Chrysomelidae) al aumentar las poblaciones de sus depredadores. En el caso de cultivos de la palma aceitera, Giller y Fairhurst (2012) en Malasia y Arango et al. (2011) en Colombia, indican que las coberturas de leguminosas reducen la incidencia de plagas $\mathrm{y}$ de insectos vectores de importantes enfermedades, ya que este tipo de coberturas controla el desarrollo de malezas de gramíneas, en las cuales estos insectos pasan parte de su ciclo de vida. Al respecto, hay que mencionar que en estado larval, las especies de Rabdopterus se alimentan de las raíces más finas de gramíneas (Muñoz 2002, Arguedas et al. 2013).

A pesar de que los cultivos de cobertura evaluados presentaron índices promedio de severidad menores que tratamientos como control manual o control químico, no se presentaron diferencias significativas entre tratamientos, por lo que la selección del mejor método de control de arvenses dependerá de factores como el costo y la respuesta de los árboles en términos de crecimiento ante los mismos.

\section{CONCLUSIONES}

Se encontraron a O.tectonae y Rhabdopterus sp. como las plagas objetivo de la plantación coincidentemente con los principales problemas fitosanitarios encontrados en plantaciones jóvenes en otros estudios.

Se encontraron diferencias significativas entre fechas de evaluación para las variables Severidad O. tectonae (MCS y MCI) e Incidencia de O. tectonae (MCS) y Severidad de Rhabdopterus sp.; pero no se encontraron diferencias entre tratamientos, lo que indica que los cultivos de 
cobertura no afectaron la incidencia y la severidad de las 2 plagas más dañinas encontradas.

No hubo diferencias entre la Incidencia y la Severidad de $O$. tectonae con respecto a la Mitad de la Copa Superior (MCS) y la Mitad de la Copa Inferior (MCI), por lo que no se recomienda dividirla de esta forma para futuras evaluaciones.

Con respecto a la distribución por categorías de severidad, para $O$. tectonae en MCS, a excepción del Control Manual, todos los individuos enfermos se encuentran en la categoría A; en MCI sí hay individuos en otras categorías, pero siempre predominan aquellos que se encuentran en la A. Para Rhabdopterus sp., también hay una gran incidencia de individuos en la Categoría A, lo que puede considerarse que las severidades son leves.

\section{AGRADECIMIENTOS}

Los autores desean agradecer a la Vicerrectoría de Investigación y Extensión del Instituto Tecnológico de Costa Rica por el financiamiento de este proyecto y a la empresa American Reforestation Company por su apoyo y a las estudiantes Paola Hernández y Cinthia Ramírez.

\section{LITERATURA CITADA}

Adams, P; Beadle, C; Mendham, N; Smethurst, J. 2003. The impact timing and duration of grass control of a young Eucalyptus globulus Labill. Plantation. New Forests: 26(2):147-165.

Arango, M; Sierra, L; Aldana, R; Martínez, G. 2011. Efecto de la aplicación de insecticidas y herbicidas en el desarrollo de la Marchitez letal de la palma de aceite en el Bajo Upía, Casanare. Palmas 32(1):11-23.

Arauz, F. 1998. Fitopatología: un análisis agroecológico. San José, CR, EUCR. 469 p.

Arguedas, M. 2004. La roya de la teca Olivea tectonae (Rac.): consideraciones sobre su presencia en Panamá y Costa Rica (en línea). Revista Forestal Mesoamericana Kurú 1(1):96-102. Consultado 7 mar. 2018. Disponible en http://revistas.tec.ac.cr/ index.php/kuru/article/view/600/2818

Arguedas, M; Cannon, P; Wingfield, M; Montenegro, F. 2013. Principales riesgos fitosanitarios en las plantaciones de teca. In de Camino R. y Morales JP. (eds.). Las plantaciones de teca en América Latina: Mitos y realidades. Turrialba, Costa Rica: FAO-CATIE. Serie Técnica. Informe Técnico $N^{\circ}$ 397. p. 134-157.

Arguedas, M; Murillo, O; Ayuso, F; Madrigal, O. 2006. Variación en la resistencia de clones de teca (Tectona grandis L.f.) ante la infección de la roya (Olivea tectonae Rac.) en Costa Rica (en línea). Revista Forestal Mesoamericana Kurú 2(6):1524. Consultado 12 mar. 2018 Disponible en http:// revistas.tec.ac.cr/index.php/kuru/article/view/534

Bachie, O. 2011. Interdisciplinary Pest Management Potentials of Cover Cropping Systems. Tesis. Ph.D. California, United States, University of California Riverside. 133 p.

Baltodano, C. 2008. Relación entre la incidencia de la roya Olivea tectonae, el cancro Dothiorella sp. y el defoliador Rhabdopterus sp. en plantaciones de Tectona grandis L.f. y el índice de calidad de sitio en Panamá (en línea). Revista Forestal Mesoamericana Kurú 5 (13). Consultado 12 ago. 2018. Disponible en http://revistas.tec.ac.cr/index.php/kuru/article/ view/468

Boley, J; Drew, A; Andrus, R. 2009. Effects of active pasture, teak (Tectona grandis) and mixed native plantations on soil chemistry in Costa Rica (en línea). Forest Ecology and Management 257: 22542261. Consultado 7 mar. 2018. Disponible en https:// doi.org/10.1016/j.foreco.2009.02.035

Bone, N; Thomson, LJ; Ridland, P; Cole, P; Hoffmann, A. 2009. Cover crops in Victorian apple orchards: effects on production, natural enemies and pests across a season. Crop Protection 28:675-683.

CABI (Centre for Agriculture and Biosciences International, UK). 2017. Invasive Species Compendium. Olivea tectonae (teak rust) (en línea). Wallingford, United Kingdom: CAB International. Consultado $28 \mathrm{feb}$. 2018. Disponible en https://www.cabi.org/isc/ datasheet $/ 37304$

Cabral, PG; Capucho, AS; Pereira, OL; Maciel-Zambolim, E; Freitas, RL; Zambolim, L. 2010. First report of teak leaf rust disease caused by Olivea tectonae in Brazil. Australasian Plant Disease Notes 5(1):113-114.

Cibrián, D; Arguedas, M. 2007. Roya de la teca Olivea tectonae (T.S. Ramakr. \& K. Ramakr.) Thirum. (Uredinales, Chaconiaceae). In Cibrián, D. (ed.). Enfermedades Forestales en México. Chapingo, México, Universidad Autónoma de Chapingo. p. 206-207.

Couto, A; Alves, F; Gonçalves, R; Coelho, R. 2007. Isolamiento de Fungus Fitopatoênicos. In Couto, A; Gonçalves, R. (eds.). Métodos em Fitopatologia. Viçosa, Brasil. Universidade de Viçosa. p. 53-91.

Danne, A; Thomson, LJ; Sharley, DJ; Penfold, CM; Hoffmann, A. 2010. Effects of native grass cover crops on beneficial and pest invertebrates in Australian vineyards. Environmental Entomology 39(3):970-978. 
De Camino, R; Morales, JP. 2013. La teca em América Latina. In Las plantaciones de teca en América Latina: Mitos y realidades. Turrialba, Costa Rica. Serie Técnica. Informe Técnico $N^{\circ}$ 397. p. 30-41.

EPPO (European and Mediterranean Plant Protection Organization). 2005. Teak rust (Olivea tectonae) is spreading in America. Paris, France, EPPO. Reporting Service 8. 10 p.

FSC (Forest Stewardship Council, DEU). 2015. Lista de pesticidas "altamente peligrosos del FSC" (en línea). FSC-STD-30-001a ES. Bonn, DEU, FSC International Center. Cosultado 22 mar. 2018. Disponible en https://ic.fsc.org/file-download. fsc-std-30-001a-es-lista-de-pesticidas-altamentepeligrosos.a-7016.pdf

Garau, A; Ghersa, C; Lemcoff, J; Barañao, J. 2009. Weeds in Eucalyptus globulus subsp. Maidenii (F. Muell) Establishment: Effects of Competition on Sapling Growth and Survivorship. New Forests 37:251-264.

Giller, K; Fairhurst, T. 2012. Plantas leguminosas de cobertura. In Fairhurst, T; Hardter, R. (eds.). Palma de Aceite: Manejo para Rendimientos Altos y Sostenibles. Georgia, USA, International Plant Nutritional Institute (IPNI) y International Potash Institute (IPI). p. 173-184.

Guevara, M. 2011. Productividad y costos de la chapea mecanizada en plantaciones jóvenes de Acacia mangium en la zona norte de Costa Rica. (en línea). Revista Forestal Mesoamericana Kurú 8(20):27-30. Consultado 7 mar. 2018. Disponible en http://revistas. tec.ac.cr/index.php/kuru/article/view/376/309

INEC (Instituto Nacional de Estadística y Censos). 2015. VI Censo Nacional Agropecuario: Cultivo Agrícolas, Forestales y Ornamentales. San José, Costa Rica. 285 p.

Jabran, K; Mahajan, G; Sardana, V; Chauhan, BS. 2015. Allelopathy for weed control in agricultural systems. Crop Protection 72(0):57-65.

Keogh, M. 2013. La teca y su importancia económica a nivel mundial. In de Camino, R; Morales, JP (eds.). Las plantaciones de teca en América Latina: Mitos y realidades. Turrialba, Costa Rica: FAO-CATIE. Serie Técnica. Informe Técnico No 397:8-28.

Kollert, W; Kleine, M. 2017. The Global Teak Study. Analysis, Evaluation and Future Potential of Teak Resources. IUFRO World Series. v 36. 108 p.

Ladrach, W. 2010. Manejo práctico de plantaciones forestales en el trópico y subtrópico. Cartago, CR, Editorial Tecnológica. $660 \mathrm{p}$.
Lundgren, JG; Fergen, JK. 2011. Enhancing predation of a subterranean insect pest: a conservation benefit of winter vegetation in agroecosystems. Appl. Soil Ecol. 51:9-16.

Matarrita, L; Sandoval, J; Arguedas, M. 2006 Prevalencia de la roya Olivea tectonae (Rac.) de la teca (Tectona grandis L.f.) (en línea). Revista Forestal Mesoamericana Kurú 3(9):1223. Consultado 7 mar. 2018. Disponible en http:// www.itcr.ac.cr/revistakuru/anteriores/anterior9/ Articulo\%202.htm

Mesquita, JB; dos Santos, ITBF; Ribeiro, GT; Campos, MJ 2016. Ocorrência de ferrugem (Olivea neotectonae) em plantas de teca no estado de Sergipe. Summa Phytopathologica 42(3):278-279.

Montagnini, F. 2004. Plantaciones forestales con especies nativas: una alternativa para la producción de madera y la provisión de servicios ambientales. Recursos Naturales y Ambiente 43:28-35.

Moya, R; Bond, B; Quesada-Pineda, H. 2014. A review of heartwood properties of Tectona grandis trees from fast-growth plantations. Wood Sci. Technol. 48(2):411-433.

Muñoz, R. 2002. Estudios básicos del defoliador Rhabdopterus sp. en plantaciones de teca (Tectona grandis) de Flor y Fauna S.A. y Brinkman y Asociados Reforestadores de Centroamérica S.A. Informe de Práctica de Especialidad. Cartago, CR, ITCR. $100 \mathrm{p}$.

Pérez, M; López, MO; Martí, O. 2008. Olivea tectonae, leaf rust of teak, occurs in Cuba. New Disease Reports 17. p. 32.

SAS-Institute Inc. 2000. SAS/STAT User's Guide, Version 8. SAS Institute Inc., Cary, North Carolina, United States. 782 p.

Sales, NIS; Leão, EU; Correia, LCMDA; Siqueira, CDA; Santos, GRD. 2017. Temporal progress of teak rust in a tropical area of Tocantins State, Brazil. Acta Amazonica 47(3):277-280.

Schipanski, ME; Barbercheck, M; Douglas, MR; Finney, DM; Haider, K; Kaye, JP; Kemanian, AR; Mortense, DA; Ryan, MR; Tooker, J; White, C. 2014. A framework for evaluating ecosystem services provided by cover crops in agroecosystems. Agricultural Systems 125:12-22.

United States Department of Agriculture (USDA). 2003. Keys to Soil Taxonomy. Natural Resources Conservation Service. Washington DC, United States. 332 p.

Todos los derechos reservados. Universidad de Costa Rica. Este artículo se encuentra licenciado con Creative Commons Reconocimiento-NoComercial-SinObraDerivada 3.0 Costa Rica. Para mayor información escribir a rac.cia@ucr.ac.cr 
